\title{
Real-world disease burden and outcomes of brain metastases in EGFR mutation-positive non-small-cell lung cancer
}

Eric Nadler*,1, Janet L Espirito², Melissa Pavilack³, Bismark Baidoo² \& Ancilla Fernandes ${ }^{3}$

${ }^{1}$ Texas Oncology, Dallas, TX 75246, USA

${ }^{2}$ McKesson Life Sciences, The Woodlands, TX 77380, USA

${ }^{3}$ AstraZeneca, Gaithersburg, MD 20878, USA

*Author for correspondence: eric.nadler@usoncology.com

Aim: To evaluate the real-world impact of brain metastases (BM) among patients with EGFR mutationpositive (EGFRm) metastatic non-small-cell lung cancer (NSCLC). Materials \& methods: This retrospective, observational matched cohort electronic health record study assessed adults with EGFRm metastatic NSCLC with/without BM. Results: Among 402 patients split equally between both cohorts ( $\pm B M)$, the majority were Caucasian (69\%), female (65\%) and with adenocarcinoma (92\%). Overall symptom burden and ancillary support service use were higher and median overall survival from metastatic diagnosis was significantly shorter in BM patients (11.9 vs 16 months; $\mathrm{p}=0.017$ ). Conclusion: BM in EGFRm NSCLC patients can negatively impact clinical outcomes. New targeted therapies that can penetrate the blood-brain barrier should be considered for treating these patients.

\section{Graphical abstract:}

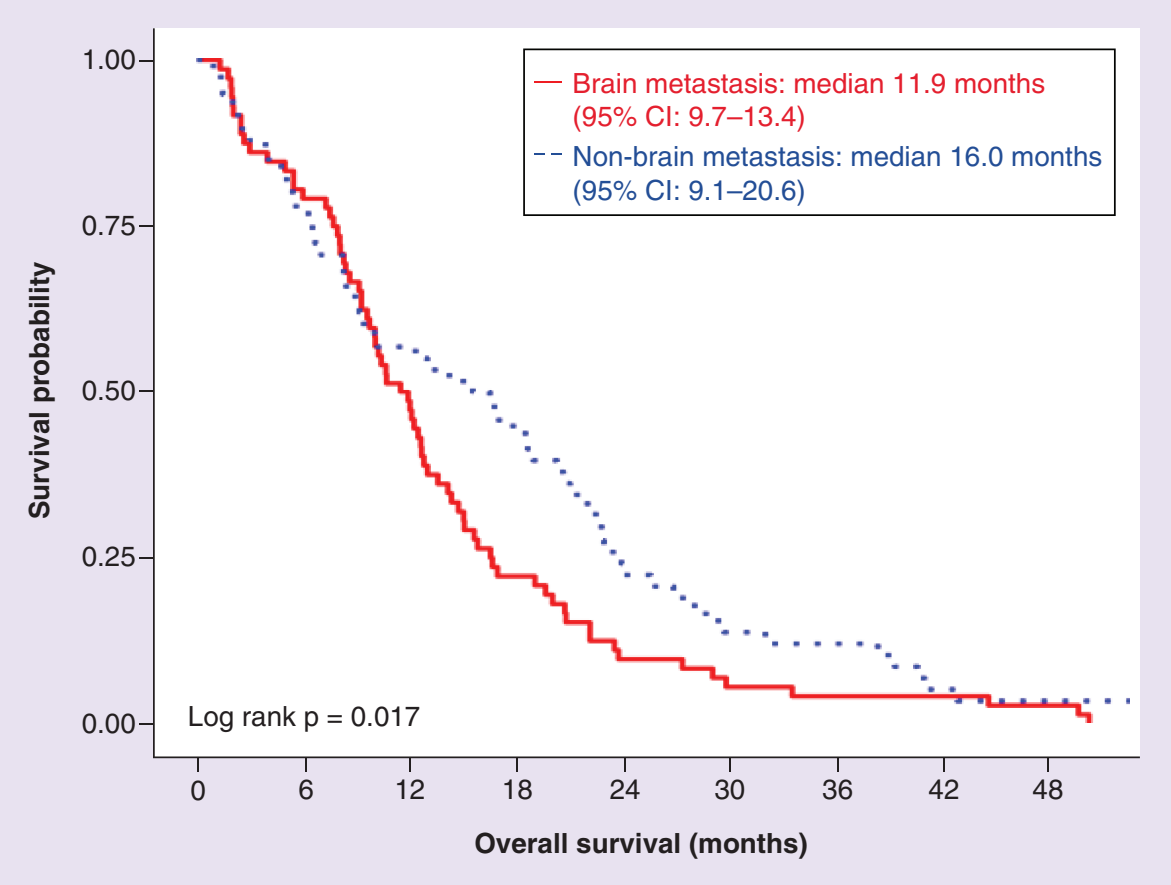

First draft submitted: 31 March 2020; Accepted for publication: 15 May 2020; Published online: 4 June 2020

Keywords: brain metastases • CNS • EGFR • EGFR-TKI • non-small-cell lung cancer • NSCLC • osimertinib • realworld 
Lung cancer is the most common cancer worldwide [1]. In the USA, over 200,000 new cases are diagnosed annually and it is the leading cause of cancer-related mortality for both men and women [2]. Most patients are diagnosed at a late stage, with approximately $70 \%$ of new diagnoses having locally advanced or metastatic disease, which can include specifically brain or central nervous system (CNS) metastases [3].

The incidence of brain metastasis is increasing, potentially due to better treatment and prolonged survival [4]. This condition can be associated with significant burden of illness [5-7]. In addition, the brain is a common site of metastasis, occurring in $17-65 \%$ of patients with primary lung cancer [8]. The brain can be a safe harbor for tumor growth even when there is good visceral control, due to the limited ability of systemic therapies to cross the blood-brain barrier $[9,10]$.

Molecular understanding of non-small-cell lung cancer (NSCLC) in the past few years has revolutionized the treatment of this type of lung cancer, with the introduction of targeted therapies which are less toxic than conventional chemotherapies [11,12]. Biomarkers and associated targeted therapies have improved diagnoses and prognoses for many patients with NSCLC [13].

EGFR mutations are observed in approximately $14 \%$ of patients with NSCLC [14]. The EGFR mutationpositive $(E G F R \mathrm{~m})$ subtype is associated with specific pathologic features. The prevalence of brain metastases in $E G F R \mathrm{~m}$ patients specifically is not well known and the incidence may increase as a consequence of improved survival with emerging therapies [15].

The objective of this study was to evaluate patient characteristics, symptom burden, support care services and outcomes, including time to treatment failure (TTF) and overall survival (OS), in patients with EGFRm metastatic NSCLC, with and without brain metastases, in a real-world community oncology setting.

\section{Materials \& methods}

\section{Study design \& data sources}

This was a retrospective, observational matched cohort electronic health record (EHR) study of patients who received care within a US Oncology Network clinic between January 12014 and July 31 2016, with follow-up through March 31 2017. Institutional Review Board approval was obtained for the study. Patients with EGFRm metastatic NSCLC with and without brain metastases were identified from the iKnowMed EHR and matched 1:1 by 10-year age bands and sex. The US Oncology Network is affiliated with approximately 60 community oncology practices across 25 states, with approximately 1400 physicians. Data were initially collected via programmatic queries of the iKnowMed EHR system and supplemented with chart review to confirm eligibility, assign into cohorts and to collect clinical symptoms and referrals for ancillary support services. Included patients were $\geq 18$ years of age at diagnosis of NSCLC, with confirmed EGFRm metastatic disease and at least two visits during the study period (including the follow-up period). Patients could have developed brain metastases before or after receipt of therapy. Patients enrolled in clinical trials or with other concomitant cancer diagnoses during the study period were excluded. The Social Security Death Index was used to supplement vital status from the EHR data. The index date for the cohort of patients with evidence of brain metastases was the date of diagnosis of brain metastases. The index date for the cohort of patients without evidence of brain metastases was the start date of the first treatment for their closest matched line of therapy. Brain imaging at the time of diagnosis was not required for entry into the study; however, among patients with imaging performed, timing and type of imaging used was collected. CNS symptoms and use of ancillary support services were abstracted from chart review through progress notes and use of referrals. All symptoms were abstracted regardless of reason as causality may not be able to be attributed retrospectively.

\section{Statistical analysis}

Comparative analyses between the brain metastasis and non-brain metastasis cohorts were performed using $\chi^{2} /$ Fisher's exact test (for categorical variables) and t-test/Mann-Whitney $U$ test/analysis of variance (ANOVA)/Kruskal-Wallis test (for the continuous variables), as applicable. TTF was calculated from the start of treatment to the end of treatment for any reason or censoring. In the brain metastasis cohort, treatment start was calculated from start of treatment following the diagnosis of brain metastasis, and in patients without brain metastasis, from the start of the equivalent line of therapy to understand treatment durations at similar points in the trajectory of care. OS for both cohorts was calculated from the date of metastatic diagnosis and patients alive at the end of the study or lost to follow-up were censored. Kaplan-Meier methods were used for TTF and OS estimates 


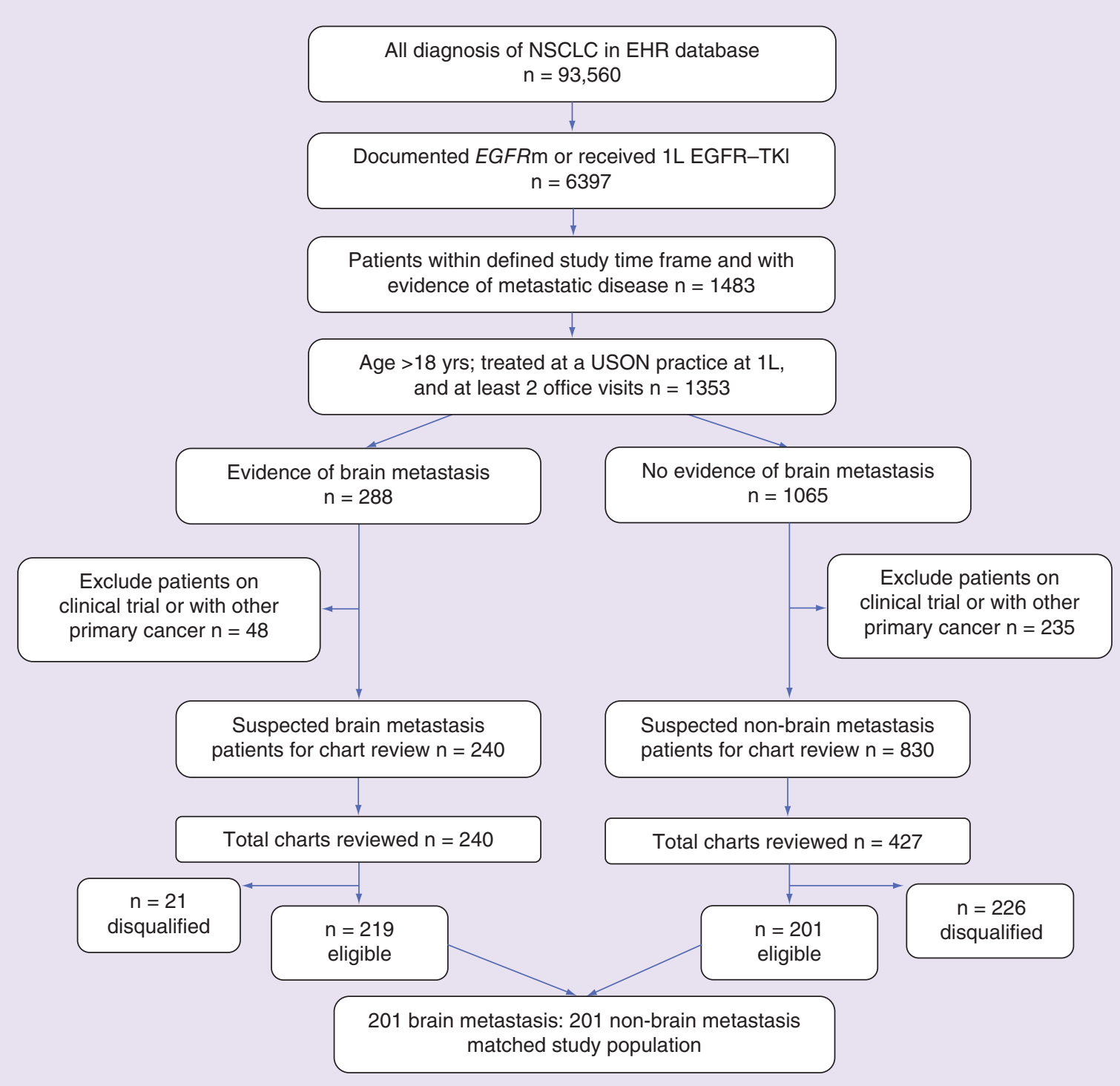

Figure 1. Patient selection.

1L: First line; EGFRm: EGFR mutation positive; EHR: Electronic health record; NSCLC: Non-small-cell lung cancer; TKI: Tyrosine kinase inhibitor; USON: US Oncology Network.

and log-rank tests were used to determine differences. Statistical significance was defined as p-values $<0.05$. The analyses were conducted using SAS ${ }^{\circledR}$ (version 9.4, SAS Institute Inc., NC, USA).

\section{Results}

\section{Patient characteristics}

Over 93,000 patients with NSCLC were identified from the EHR database (Figure 1). Approximately 7\% were documented as EGFRm, as identified from structured EHR data collected, or as having received a first-line EGFR tyrosine kinase inhibitor (TKI) for which EGFR status would be confirmed from chart review. After application of other structured data eligibility criteria, 667 patient charts underwent chart review to confirm eligibility and obtain at least the 200 patients for each cohort. Among patients initially identified as having no brain metastases, a common reason for disqualification was the discovery of brain metastasis during chart review, in at least $15 \%$ of patients. After cohort matching, 402 final patients were included, 201 patients with brain metastases and 201 patients without brain metastases. 
The patient characteristics were similar across both cohorts. Overall, the majority of patients were Caucasian (68.7\%), female (65.2\%), never smokers (41.5\%) and with adenocarcinoma histology (92.0\%). Patients ranged from stage I to IV disease at initial diagnosis; however, most patients were initially diagnosed with stage IV disease (75.1\%) and those with earlier stages at diagnosis were later metastatic. However, patients with brain metastases were significantly younger than those without (median age: 70 vs 77 years; $\mathrm{p}=0.0004$ ) and a higher proportion of patients with brain metastasis were initially diagnosed with stage IV disease (81.6\%) than those without (68.7\%; $\mathrm{p}=0.04$; Table 1). Among the patients with brain metastases, baseline brain imaging within 30 days of metastatic diagnosis occurred in $86 \%$ of patients, demonstrating that most patients did have brain imaging around the time of metastatic diagnosis. The predominant imaging method used was MRI in $80.1 \%$ of patients, CT scan in $6.5 \%$ and unknown in $13.4 \%$. Most patients with brain metastases (67\%) had CNS symptoms present at the time of diagnosis of brain metastases, although approximately a third did not have CNS symptoms noted.

\section{Clinical symptoms \& support services}

CNS symptoms were observed in both cohorts, though rates for most were observed to be significantly higher in patients with brain metastases. Symptoms occurring in at least $10 \%$ of patients with brain metastases but in $<10 \%$ in patients without brain metastases included seizures, speech problems, focal neurologic deficits, drowsiness, problems with memory and altered mental status (Table 2). Symptoms occurring in $>10 \%$ of patients in both the non-brain metastases and brain metastases cohorts included nausea, vomiting, vision disorder, headache and balance/mobility symptoms. Patients with brain metastases had significantly greater use of home healthcare, nutrition therapy, physical therapy, rehabilitation and social work services than patients without brain metastases (Table 3).

\section{Clinical outcomes}

Most patients were receiving first-line treatment at study entry, 93.0\% $(\mathrm{n}=187)$ in the brain metastasis cohort and $96.5 \%(n=194)$ of patients without brain metastasis. Similar proportions of patients were treated with a firstgeneration EGFR-TKI in the two cohorts: $78.1 \%(\mathrm{n}=157)$ of patients with brain metastasis and $73.6 \%(\mathrm{n}=148)$ of patients without brain metastasis. Median TTF was similar between both cohorts; 10.9 months (95\% CI: 9.5-12.0) for patients with brain metastasis and 10.4 months (95\% CI: 8.9-12.2) for patients without; $\mathrm{p}=0.5184$ (Figure 2). However, median OS from metastatic diagnosis was significantly lower in patients with brain metastasis than patients without (11.9 months [95\% CI: 9.7-13.4] vs 16.0 months [95\% CI: 9.1-20.6], respectively; $\mathrm{p}=0.017$; Figure 3).

\section{Discussion}

Consistent with previous global studies of known characteristics of patients with EGFRm NSCLC, most patients in this study were female never smokers with adenocarcinoma histology [16]. However, unlike such global studies, where a higher proportion of Asian patients typically have EGFRm status, most patients in this study were Caucasian.

Ongoing research is being conducted to evaluate the association between EGFR mutation and CNS metastases and impact on outcomes. In one study by Stanic et al., of 168 lung adenocarcinoma patients with CNS metastases, 28\% ( $\mathrm{n}=47)$ of the patients had an EGFR mutation [4]. The time from brain metastases to death in EGFRm patients with brain metastases at diagnosis was 12.6 months compared with 6.8 months in patients without an EGFR mutation $(\mathrm{p}=0.005)$.

In another study of 1522 patients with NSCLC, 30\% of patients $(\mathrm{n}=452)$ were EGFRm [17]. Among those with the EGFR mutation, 21\% ( $\mathrm{n}=93)$ had brain metastases and 79\% $(\mathrm{n}=359)$ did not. Regardless of EGFR mutation status, median OS was significantly shorter in those patients with brain metastases than those without brain metastases ( 15 vs 20.6 months; $\mathrm{p}=0.02$ ). However, in the $E G F R \mathrm{~m}$ patients specifically, there was no significant difference in median OS from the time of initial diagnosis in those patients with and without brain metastases (20.8 vs 25.1 months; $\mathrm{p}=0.11$ ).

In both of these previous studies, the number of patients with both an EGFR mutation and brain metastasis were small ( $\mathrm{n}=47$ and $\mathrm{n}=93$ in either study). Given the limited information, our study helps establish some baseline understanding of symptoms and outcomes in patients prior to the availability of newer therapies and may allow us to understand how outcomes have changed over time in these patients, specifically with the use of new therapies. In our study, all patients were EGFRm and the sample size of patients with brain metastases was larger, with 201 patients. We observed the median TTF to be similar in patients with and without metastases and this 


\begin{tabular}{|c|c|c|c|c|}
\hline Variable & $\begin{array}{l}\text { Overall } \\
(n=402)\end{array}$ & $\begin{array}{l}\text { Brain metastasis } \\
(n=201)\end{array}$ & $\begin{array}{l}\text { Non-brain metastasis } \\
(n=201)\end{array}$ & p-value ${ }^{\dagger}$ \\
\hline \multicolumn{5}{|l|}{ Age (years } \\
\hline Median & 73.0 & 70.0 & 77.0 & \multirow[t]{2}{*}{0.0004} \\
\hline Range (min-max) & $39->90$ & $39->90$ & $43.0->90$ & \\
\hline \multicolumn{5}{|l|}{ Gender } \\
\hline Female & $262(65.2)$ & $131(65.2)$ & $131(65.2)$ & 0.7549 \\
\hline Male & $140(34.8)$ & $70(34.8)$ & $70(34.8)$ & \\
\hline \multicolumn{5}{|l|}{ Race } \\
\hline African-American & $31(7.7)$ & $15(7.5)$ & $16(8.0)$ & 0.4139 \\
\hline Caucasian & $276(68.7)$ & $132(65.7)$ & $144(71.6)$ & \\
\hline Other & $50(12.4)$ & $29(14.4)$ & $21(10.4)$ & \\
\hline Unknown & $45(11.2)$ & $25(12.4)$ & $20(10.0)$ & \\
\hline \multicolumn{5}{|l|}{ Smoking status } \\
\hline Current & $19(4.7)$ & $9(4.5)$ & $10(5.0)$ & 0.7076 \\
\hline Former & $121(30.1)$ & $49(24.4)$ & $72(35.8)$ & \\
\hline Never & $167(41.5)$ & $75(37.3)$ & $92(45.8)$ & \\
\hline Unknown & $95(23.6)$ & $68(33.8)$ & $27(13.4)$ & \\
\hline \multicolumn{5}{|l|}{ EGFR T790M mutation status } \\
\hline Negative & $51(12.7)$ & $29(14.4)$ & $22(10.9)$ & 0.9834 \\
\hline Positive & $60(14.9)$ & 34 (16.9) & $26(12.9)$ & \\
\hline Unknown & $291(72.4)$ & $138(68.7)$ & $153(76.1)$ & \\
\hline \multicolumn{5}{|l|}{ Stage at initial NSCLC diagnosis } \\
\hline IA & $12(3.0)$ & $2(1.0)$ & $10(5.0)$ & 0.04 \\
\hline IB & $14(3.5)$ & $9(4.5)$ & $5(2.5)$ & \\
\hline IIA & $12(3.0)$ & $4(2.0)$ & $8(4.0)$ & \\
\hline IIB & $5(1.2)$ & $3(1.5)$ & $2(1.0)$ & \\
\hline IIIA & $26(6.5)$ & $11(5.5)$ & $15(7.5)$ & \\
\hline IIIB & $9(2.2)$ & $3(1.5)$ & $6(3.0)$ & \\
\hline IV & $302(75.1)$ & $164(81.6)$ & $138(68.7)$ & \\
\hline Not documented & $6(1.5)$ & $1(0.5)$ & $5(2.5)$ & \\
\hline Unknown & $16(4.0)$ & $4(2.0)$ & $12(6.0)$ & \\
\hline \multicolumn{5}{|l|}{ Histology } \\
\hline Adenocarcinoma & $370(92.0)$ & $191(95.0)$ & $179(89.1)$ & 0.3459 \\
\hline Adenosquamous carcinoma & $7(1.7)$ & $3(1.5)$ & $4(2.0)$ & \\
\hline Bronchiolo-alveolar carcinoma & $4(1.0)$ & $1(0.5)$ & $3(1.5)$ & \\
\hline Unknown & $16(4.0)$ & $5(2.5)$ & $11(5.5)$ & \\
\hline Unspecified NSCLC & $5(1.2)$ & $1(0.5)$ & $4(2.0)$ & \\
\hline \multicolumn{5}{|c|}{ ECOG performance status at index } \\
\hline ECOG 0 & $41(10.2)$ & $20(10.0)$ & $21(10.4)$ & 0.3098 \\
\hline ECOG 1 & $206(51.2)$ & $85(42.3)$ & $121(60.2)$ & \\
\hline ECOG 2 & $36(9.0)$ & $12(6.0)$ & $24(11.9)$ & \\
\hline ECOG 3 & $3(0.7)$ & $0(0.00)$ & $3(1.5)$ & \\
\hline Unknown & $116(28.9)$ & $84(41.8)$ & $32(15.9)$ & \\
\hline \multicolumn{5}{|c|}{$\begin{array}{l}{ }^{\dagger} \text { For comparison of brain metastasis and non-brain metastasis cohorts. } \\
\text { Data are presented as n (\%) unless otherwise specified. } \\
\text { ECOG: Eastern Cooperative Oncology Group; NSCLC: Non-small-cell lung cancer. }\end{array}$} \\
\hline
\end{tabular}

may have been influenced by the observation that most patients were receiving first-line of therapy at study entry; however, median OS from time of metastatic disease was significantly shorter for patients with brain metastases at 11.9 months compared with those without (16.0 months). 
Table 2. Clinical symptoms in patients with and without brain metastasis.

\begin{tabular}{|c|c|c|c|c|}
\hline Symptoms & $\begin{array}{l}\text { Overall } \\
(n=402)\end{array}$ & $\begin{array}{l}\text { Brain metastasis } \\
(\mathrm{n}=201)\end{array}$ & $\begin{array}{l}\text { Non-brain metastasis } \\
(n=201)\end{array}$ & p-value ${ }^{\dagger}$ \\
\hline Fatigue & $288(71.6)$ & $164(81.6)$ & $124(61.7)$ & $<0.0001$ \\
\hline Depression & $104(25.9)$ & $65(32.3)$ & $39(19.4)$ & 0.0031 \\
\hline Seizure & $35(8.7)$ & $34(16.9)$ & $1(0.5)$ & $<0.0001$ \\
\hline Speech problems & $38(9.5)$ & $34(16.9)$ & $4(2.0)$ & $<0.0001$ \\
\hline Stroke & $14(3.5)$ & $7(3.5)$ & $7(3.5)$ & 1 \\
\hline Vision disorder & 77 (19.2) & $53(26.4)$ & $24(11.9)$ & 0.0002 \\
\hline Vomiting & $101(25.1)$ & $70(34.8)$ & $31(15.4)$ & $<0.0001$ \\
\hline Cognitive impairment & $27(6.7)$ & $19(9.5)$ & $8(4.0)$ & 0.0284 \\
\hline Pain or numbness & $282(70.1)$ & $148(73.6)$ & $134(66.7)$ & 0.127 \\
\hline Balance/mobility & $111(27.6)$ & $73(36.3)$ & $38(18.9)$ & $<0.0001$ \\
\hline Changes in mood/personality & $17(4.2)$ & $8(4.0)$ & $9(4.5)$ & 0.8043 \\
\hline Nausea & $198(49.3)$ & $123(61.2)$ & 75 (37.3) & $<0.0001$ \\
\hline Focal neurologic deficits & 78 (19.4) & $66(32.8)$ & $12(6.0)$ & $<0.0001$ \\
\hline Drowsiness & $65(16.2)$ & $46(22.9)$ & $19(9.5)$ & 0.0003 \\
\hline Headache & 159 (39.6) & $117(58.2)$ & $42(20.9)$ & $<0.0001$ \\
\hline Problems with memory & 75 (18.7) & $61(30.3)$ & $14(7.0)$ & $<0.0001$ \\
\hline Altered mental status & $51(12.7)$ & $33(16.4)$ & $18(9.0)$ & 0.0246 \\
\hline Anxiety & $144(35.8)$ & $83(41.3)$ & $61(30.3)$ & 0.0221 \\
\hline
\end{tabular}

\section{Table 3. Ancillary support service utilization in patients with and without brain metastasis.}

\begin{tabular}{|c|c|c|c|c|}
\hline Variables & $\begin{array}{l}\text { Overall } \\
(n=402)\end{array}$ & $\begin{array}{l}\text { Brain metastasis } \\
(n=201)\end{array}$ & $\begin{array}{l}\text { Non-brain metastasis } \\
(n=201)\end{array}$ & p-value ${ }^{\dagger}$ \\
\hline Home healthcare & $95(23.6)$ & $63(31.3)$ & $32(15.9)$ & 0.0003 \\
\hline Nutrition therapy & $54(13.4)$ & 35 (17.4) & 19 (9.5) & 0.0193 \\
\hline Mental health/psych evaluation/treatment & $19(4.7)$ & $8(4.0)$ & $11(5.5)$ & 0.4807 \\
\hline Physical therapy & $71(17.7)$ & $54(26.9)$ & $17(8.5)$ & $<0.0001$ \\
\hline Rehabilitation & $53(13.2)$ & $34(16.9)$ & $19(9.5)$ & 0.027 \\
\hline Social work/services & $60(14.9)$ & $38(18.9)$ & $22(10.9)$ & 0.0251 \\
\hline Emergency room visit and hospitalizations post index & $155(38.6)$ & $87(43.3)$ & $68(33.8)$ & 0.0515 \\
\hline
\end{tabular}

Not surprisingly, symptom burden and use of ancillary support services were observed to be higher for patients with brain metastases. Notably, however, many of the same symptoms were present in both cohorts, potentially suggesting the presence of micrometastases not visible by scan. Additionally, some symptoms may have been associated with the extra-cranial disease and treatment and not the brain metastases. This reflects the ongoing need to be able to identify and provide effective treatments among patients with advanced EGFRm NSCLC. As new therapies prolong survival for EGFRm patients, a greater understanding of treatment-related burden of illness and longer-term complications is needed. New therapies to treat brain metastases or better management strategies for monitoring and treating at-risk patients, would address an important unmet medical need.

Treatment of brain metastasis can be multidisciplinary, including combinations of surgery, radiation and/or systemic therapies that may cross the blood-brain barrier. There is increasing evidence supporting the intracranial activity of EGFR-TKIs in patients with NSCLC and brain metastasis. One published review examined the use of the first-generation EGFR-TKIs gefitinib and erlotinib [9]. In the eight Phase II clinical trials included in the review, the intracranial response rates were $27-32 \%$ in unselected patients, $43-74 \%$ in patients with demographics associated with the EGFR mutation genotype such as Asian, never smoker and adenocarcinoma and 56-89\% in patients with the EGFR mutation. 


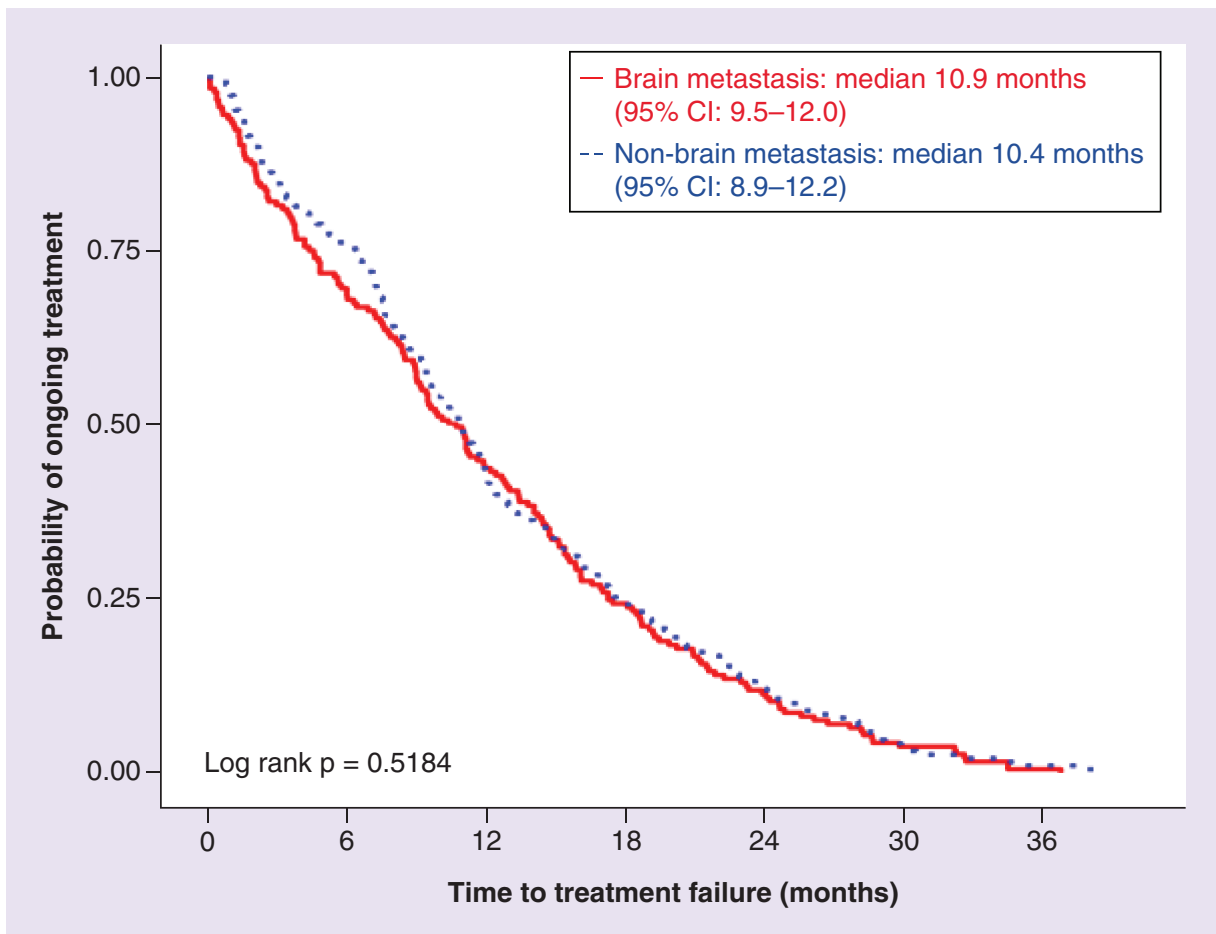

Figure 2. Median time to treatment failure in patients with and without brain metastasis.

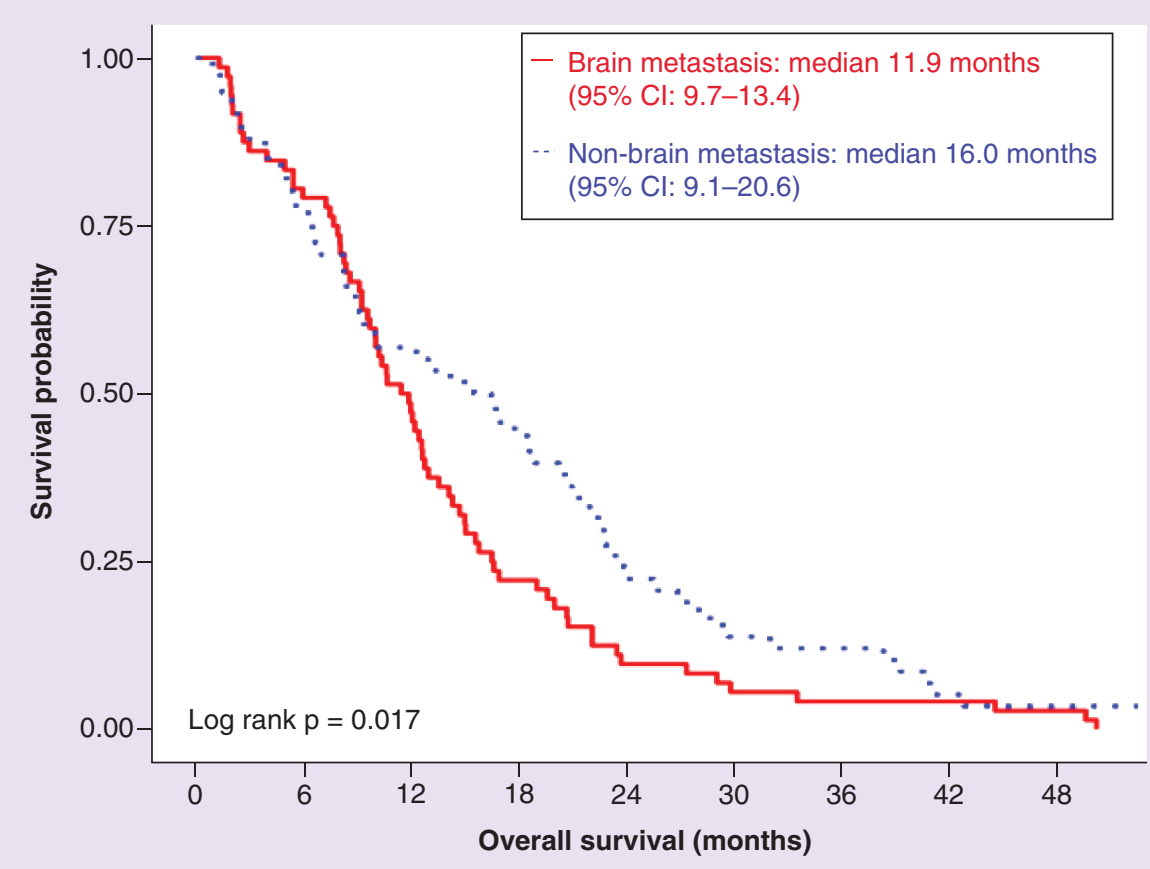

Figure 3. Median overall survival from metastatic diagnosis in patients with and without brain metastasis.

The CNS activity of newer generation EGFR-TKIs have been shown in preclinical studies [18-21]. Osimertinib, a third-generation, irreversible, oral, EGFR-TKI that potently and selectively inhibits both EGFR-TKI sensitizing mutations and the EGFR T790M resistance mutation, has been shown to achieve significant exposure in the brain compared with other EGFR-TKIs [18,19]. 
In our study, the majority of patients had received first-line treatment with the first-generation EGFR-TKI erlotinib at time of study entry, with no patients receiving a third-generation EGFR-TKI, as this treatment was not approved during the course of this study. Recently, the Phase III FLAURA trial compared the third-generation EGFR-TKI osimertinib with either erlotinib or gefitinib as first-line treatment of EGFRm advanced NSCLC [21]. Approximately $20 \%$ of patients in the trial had CNS metastases and fewer CNS progression events were observed in patients treated with osimertinib (6\%) versus those receiving erlotinib or gefitinib (15\%) [22]. Final OS results from FLAURA demonstrated significantly longer OS in those who received osimertinib versus comparator EGFR-TKI, with a $20 \%$ lower risk of death (median OS of 38.6 months, 95\% CI: 34.5-41.8; vs 31.8 months, 95\% CI: 26.6-36.0, respectively; $\mathrm{p}=0.046)$ [23].

A recent meta-analysis of 4373 patients who had NSCLC with brain metastases suggests that EGFR mutations are associated with significantly improved OS compared with $E G F R$ wild-type (hazard ratio: 0.73 ; 95\% CI: $0.54-$ 0.99; $\mathrm{p}=0.045$ ) [24]. This may be due to better CNS efficacy of early-generation EGFR-TKIs than chemotherapy; nonetheless, the occurrence of CNS metastases in patients with EGFRm NSCLC remains high [25,26]. Further research to understand the impact of specific treatments used in the EGFRm landscape on symptom burden and survival outcomes in patients with brain metastases is warranted.

\section{Strengths/limitations}

The strengths of this study lie in the clinically rich real-world data used to assess patient symptoms and outcomes for patients with EGFRm NSCLC with and without brain metastases in the community-based setting. Limitations include the retrospective, observational nature of the study, including the potential for missing data and documentation errors in the EHR. The iKnowMed EHR contains information on patients when they are seen by their physicians or as reported to their physician and recorded in the EHR. Therefore, patient treatment history outside the US Oncology Network may not be well captured. Referrals for ancillary services were captured; however, volume and frequency of use were not. Additionally, this may have been under-captured if patients received these services outside of the network.

\section{Conclusion}

These data demonstrate an unmet treatment need for patients with EGFRm metastatic NSCLC with brain metastasis. Given recent developments in the treatment landscape, future research should explore how new targeted therapies, such as third-generation EGFR-TKIs, impact clinical outcomes among these patients and how CNS burden changes in the real world once third-generation EGFR-TKIs are used more extensively.

\section{Summary points}

- Approximately $70 \%$ of lung cancer cases are diagnosed at late-stage, either locally advanced or metastatic disease; the brain is a common site of metastasis in non-small-cell lung cancer (NSCLC) and is often a safe harbor for tumor growth, as most therapies do not cross the blood-brain barrier.

- Approximately $14 \%$ of patients with NSCLC are EGFR mutation-positive; however, the prevalence of brain metastases in these patients specifically is not well known.

- This retrospective, observational matched cohort study compared patient characteristics, disease burden (including symptoms and support care services) and outcomes in a real-world community-based setting in patients with advanced EGFR mutation-positive NSCLC with and without brain metastases.

- After cohort matching, 402 final patients were included in this study, 201 patients with brain metastases and 201 patients without brain metastases.

- Patients with brain metastases were significantly younger and had a higher proportion of initial diagnosis at stage IV disease than those without brain metastases.

- Central nervous system (CNS) symptoms were present in patients with and without brain metastasis, although occurrence was higher in patients with brain metastasis.

- Rates of most ancillary support service use were higher in patients with brain metastases compared with those without brain metastasis.

- Furthermore, median overall survival from metastatic diagnosis was shorter in patients with brain metastasis than patients without; therefore, treating brain metastases remains an important unmet medical need for these patients. 


\section{Author contributions}

The authors were fully responsible for all content and editorial decisions, involved at all stages of manuscript development and have approved the final version.

Financial \& competing interests disclosure

This study was funded by AstraZeneca, Cambridge, UK, the manufacturer of osimertinib. This research did not receive any specific grant from funding agencies in the public, commercial or not-for-profit sectors. E Nadler reports participation in speaker bureaus for Merck, Genentech and AstraZeneca and consultancy for Merck. JL Espirito and B Baidoo are McKesson employees and shareholders. M Pavilack and A Fernandes are AstraZeneca employees and shareholders. The authors have no other relevant affiliations or financial involvement with any organization or entity with a financial interest in or financial conflict with the subject matter or materials discussed in the manuscript apart from those disclosed.

The authors would like to acknowledge Charlotte Terry, of iMed Comms, Macclesfield, UK, an Ashfield Company, part of UDG Healthcare plc, for medical writing support that was funded by AstraZeneca, Cambridge, UK, in accordance with Good Publications Practice (GPP3) guidelines (http://www.ismpp.org/gpp3).

Ethical conduct of research

Institutional Review Board approval was obtained for the study.

\section{Data sharing statement}

Data underlying the findings described in this manuscript may be obtained in accordance with AstraZeneca's data sharing policy described at https://astrazenecagrouptrials.pharmacm.com/ST/Submission/Disclosure.

\section{Open access}

This work is licensed under the Attribution-NonCommercial-NoDerivatives 4.0 Unported License. To view a copy of this license, visit http://creativecommons.org/licenses/by-nc-nd/4.0/

\section{References}

Papers of special note have been highlighted as: $\bullet$ of interest; $\bullet \bullet$ of considerable interest

1. WHO. Cancer factsheet (2018). www.who.int/news-room/fact-sheets/detail/cancer

2. Siegel RL, Miller KD, Jemal A. Cancer statistics, 2019. CA Cancer J. Clin. 69(1), 7-34 (2019).

3. Molina JR, Adjei AA, Jett JR. Advances in chemotherapy of non-small-cell lung cancer. Chest 130(4), 1211-1219 (2006).

4. Stanic K, Zwitter M, Hitij NT, Kern I, Sadikov A, Cufer T. Brain metastases in lung adenocarcinoma: impact of EGFR mutation status on incidence and survival. Radiol. Oncol. 48(2), 173-183 (2014).

- Retrospective real-world study that describes the association between EGFR mutations, frequency of brain metastases and survival.

5. Peters S, Bexelius C, Munk V, Leighl N. The impact of brain metastasis on quality of life, resource utilization and survival in patients with non-small-cell lung cancer. Cancer Treat. Rev. 45, 139-162 (2016).

- Systematic review of burden of disease associated with brain metastasis from non-small-cell lung cancer.

6. Girard N, Cozzone D, de Leotoing L et al. Extra cost of brain metastases (BM) in patients with non-squamous non-small-cell lung cancer (NSCLC): a French national hospital database analysis. ESMO Open 3(6), e000414 (2018).

7. Fernandes AW, Wu B, Turner RM. Brain metastases in non-small-cell lung cancer patients on epidermal growth factor receptor tyrosine kinase inhibitors: symptom and economic burden. J. Med. Econ. 20(11), 1136-1147 (2017).

- Burden of illnesss study in non-small-cell lung cancer patients with brain metastases treated with EGFR tyrosine kinase inhibitors.

8. Fox BD, Cheung VJ, Patel AJ, Suki D, Rao G. Epidemiology of metastatic brain tumors. Neurosurg. Clin. N. Am. 22(1), 1-6, v (2011).

9. Jamal-Hanjani M, Spicer J. Epidermal growth factor receptor tyrosine kinase inhibitors in the treatment of epidermal growth factor receptor-mutant non-small-cell lung cancer metastatic to the brain. Clin. Cancer Res. 18, 938-944 (2012).

10. Wang BX, Ou W, Mao XY, Liu Z, Wu HQ, Wang SY. Impacts of EGFR mutation and EGFR-TKIs on incidence of brain metastases in advanced non-squamous NSCLC. Clin. Neurol. Neurosurg. 160, 96-100 (2017).

11. Gaughan E, Costa D. Genotype-driven therapies for non-small-cell lung cancer: focus on $E G F R, K R A S$ and $A L K$ gene abnormalities. Ther. Adv. Med. Oncol. 3, 335-348 (2011).

12. Cheng H, Xu X, Costa D, Al E. Molecular testing in lung cancer: the time is now. Curr. Oncol. Rep. 12, 335-348 (2010). 
13. Aisner DL, Marshall CB. Molecular pathology of non-small-cell lung cancer: a practical guide. Am. J. Clin. Pathol. 138(3), 332-346 (2012).

14. Shigematsu H, Lin L, Takahashi T et al. Clinical and biological features associated with epidermal growth factor receptor gene mutations in lung cancers. J. Natl Cancer Inst. 97(5), 339-346 (2005).

15. Rangachari D, Yamaguchi N, Vanderlaan PA et al. Brain metastases in patients with $E G F R$-mutated or $A L K$-rearranged non-small-cell lung cancers. Lung Cancer 88(1), 108-111 (2015).

16. Garinet S, Laurent-Puig P, Blons H, Oudart JB. Current and future molecular testing in NSCLC, what can we expect from new sequencing technologies? J. Clin. Med. 7(6), 144 (2018).

17. Bhatt VR, D'Souza SP, Smith LM et al. Epidermal growth factor receptor mutational status and brain metastases in non-small-cell lung cancer. J. Glob. Oncol. 3(3), 208-217 (2016).

- Large retrospective study evaluating incidence of EGFR mutations in patients with brain metastasis

18. Colclough N, Ballard PG, Barton P et al. 64 - Preclinical comparison of the blood-brain barrier (BBB) permeability of osimertinib (AZD9291) with other irreversible next generation EGFR-TKIs. Eur. J. Cancer 69(Suppl. 1), S28 (2016).

19. Ballard P, Yates JW, Yang Z et al. Preclinical comparison of osimertinib with other EGFR-TKIs in EGFR-mutant NSCLC brain metastases models and early evidence of clinical brain metastases activity. Clin. Cancer Res. 22(20), 5130-5140 (2016).

20. Wu YL, Ahn MJ, Garassino MC et al. CNS efficacy of osimertinib in patients with T790M-positive advanced non-small-cell lung cancer: data from a randomized Phase III trial (AURA3). J. Clin. Oncol. 36(26), 2702-2709 (2018).

21. Reungwetwattana T, Nakagawa K, Cho BC et al. CNS response to osimertinib versus standard epidermal growth factor receptor tyrosine kinase inhibitors in patients with untreated EGFR-mutated advanced non-small-cell lung cancer. J. Clin. Oncol. 36(33), 3290-3297 (2018).

22. Soria JC, Ohe Y, Vansteenkiste J et al. Osimertinib in untreated EGFR-mutated advanced non-small-cell lung cancer. $N$. Engl. J. Med. 378(2), 113-125 (2018).

23. Ramalingam SS, Vansteenkiste J, Planchard D et al. Overall survival with osimertinib in untreated, EGFR-mutated advanced NSCLC. N. Engl. J. Med. 382(1), 41-50 (2020).

24. Li W, Zhao T, $\mathrm{Xu} \mathrm{H}, \mathrm{Al} \mathrm{E}$. The role of EGFR mutation as a prognostic factor in survival after diagnosis of brain metastasis in non-small-cell lung cancer: a systematic review and meta-analysis. BMC Cancer 19(1), 145 (2019).

-• Recent meta-analysis describing the relationship between EGFR mutation, brain metastasis and survival

25. Park SJ, Kim HT, Lee DH et al. Efficacy of epidermal growth factor receptor tyrosine kinase inhibitors for brain metastasis in non-small-cell lung cancer patients harboring either exon 19 or 21 mutation. Lung Cancer 77(3), 556-560 (2012).

26. Heon S, Yeap BY, Lindeman NI et al. The impact of initial gefitinib or erlotinib versus chemotherapy on central nervous system progression in advanced non-small-cell lung cancer with EGFR mutations. Clin. Cancer Res. 18(16), 4406-4414 (2012). 